\title{
PROGRESS TOWARDS MODEL-BASED ESTIMATION OF THE CARDIAC ELECTROMECHANICAL ACTIVITY FROM ECG SIGNALS AND 4D IMAGES
}

\author{
M. Sermesant ${ }^{1}$, Y. Coudière ${ }^{2}$, H. Delingette ${ }^{1}$, N. Ayache ${ }^{1}$, J. Sainte-Marie $^{3}$, \\ D. Chapelle ${ }^{5}$, F. Clément ${ }^{4}$ and M. Sorine ${ }^{7}$
}

\begin{abstract}
We present recent advances on a 3D numerical modeling of the myocardium which couples electrical and biomechanical models. The long-term objective is to simulate a realistic contraction of the heart from both electrical measurements (typically the ECG) and geometrical measurements (typically provided by medical imaging). This realistic contraction should provide useful quantitative parameters for the diagnosis and also for guiding some new forms of therapy. Our modeling is based on a multiscale analysis ranging from microscopic to macroscopic scales, and integrates a priori information on the overall geometry and on the fiber directions extracted from specific medical imaging techniques (e.g. dtMRI). The FitzHugh-Nagumo equations are solved along with a constitutive law based on the Hill-Maxwell Rheological model, on which a data assimilation analysis is done. In medical image analysis, we believe that this new generation of physics-based deformable models will be useful to provide a more robust quantitative interpretation of temporal series of cardiac images.
\end{abstract}

Résumé. Nous présentons ici nos avancées sur la modélisation du myocarde couplant des modèles électriques et biomécaniques. L'objectif à long terme est de simuler la contraction du cœur à partir de mesures ECG et d'images médicales, afin de fournir des outils d'aide au diagnostic. Notre modèle se base sur une étude multiéhelle des phénomènes et des données anatomiques extraites d'images médicales. La partieélectrique repose sur le modèle de FitzHugh-Nagumo et la partie mécanique reprend le formalisme de Hill-Maxwell avec une nouvelle loi de comportement, sur laquelle de l'assimilation de données est réalisée. En imagerie médicale, nous pensons que ce nouveau type de modèles devrait permettre une interprétation plus robuste des séquences temporelles.

\section{INTRODUCTION}

The knowledge of the heart function has greatly improved on the nanoscopic, microscopic and mesoscopic scales during the last decades, thus a global integrative work of this organ becomes conceivable [17]. It is the objective of our multidisciplinary project ICEMA ${ }^{1}$ to build a generic dynamic model of the beating heart and a procedure to automatically adjust the parameters to any specific patient from relatively easy-to-access measurements: ECGs (electrocardiograms) and time sequences of volumetric medical images [1]. Once the

\footnotetext{
${ }^{1}$ Epidaure Research Project, INRIA Sophia-Antipolis, BP 93, 06902 Sophia-Antipolis, France

2 Mathematics Laboratory, Nantes University, France

${ }^{3}$ Macs Research Project, INRIA Rocquencourt, France

${ }^{4}$ Sosso Research Project, INRIA Rocquencourt, France

${ }^{1}$ Images of the Cardiac ElectroMechanical Activity, a collaborative research action between different INRIA projects and Philips Research France, http://www-rocq.inria.fr/who/Frederique.Clement/icema.html 
generic model is adapted to a specific patient, it becomes possible to derive a set of quantitative and objective parameters useful for clinicians and physiologists.

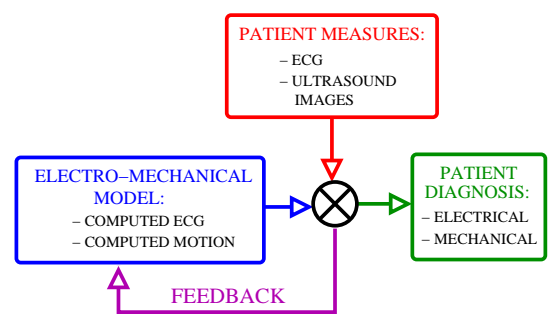

FiguRE 1. Global scheme of the long term objective.

Our approach combines a 3D numerical model of the electric wave propagation with a 3D biomechanical model of the myocardium. The two models are explicitly coupled in the simulation to generate a dynamic behavior of the heart. The model for electric wave propagation is derived from FitzHugh-Nagumo equations, while the mechanical model is based on the classical Hill-Maxwell rheological law. These models are expected to reflect on a macroscopic scale the coupling present on the cellular scale. Then, a simplified version of this electromechanical model is used to extract the heart motion from cardiac images, in the deformable model framework.

Two error functions will serve to adjust the parameters of this generic model to a specific patient: the first will compare the actual patient's ECGs with a set of ECGs computed from the simulation. The second will compare the deformation of the biomechanical model with the motion extracted from the medical images of the patient's heart. Ultimately, a feedback procedure will be used to update the parameters of the generic model from these error functions.

In this article, we develop the current stages of this on-going research. In section 1, we describe the multi-scale approach that lead to the model used. In section 2, we detail the data needed and the volumetric mesh used to carry on computations. Then in sections 3 and 4 we respectively describe the electrical and mechanical models. And section 5 describes the approach to extract the motion information from the medical image sequence.

\section{Myocardium Modeling And Control}

The cardio-vascular system can be seen as a multi-scaled hybrid system raising both modeling and control problems. The different scales concerning cardiac muscle contraction and its control are presented in the synthetic table 1 .

\begin{tabular}{|l|l|l|}
\hline scale & systems / system modeling & control / control modeling \\
\hline nanoscopic & $\begin{array}{l}\text { myosin molecules } \\
\text { Langevin equation (SDE) }\end{array}$ & $\begin{array}{l}\text { calcium ions } \\
\text { still to be designed }\end{array}$ \\
\hline microscopic & $\begin{array}{l}\text { sarcomeres } \\
\text { Huxley-like models (PDE) }\end{array}$ & $\begin{array}{l}\text { ionic currents } \\
\text { Luo-Rudy-like models (ODE) }\end{array}$ \\
\hline mesoscopic & $\begin{array}{l}\text { myofibers } \\
\text { BCS model (ODE) }\end{array}$ & $\begin{array}{l}\text { action potential } \\
\text { FitzHugh-Nagumo-like models (ODE) }\end{array}$ \\
\hline macroscopic & $\begin{array}{l}\text { myocardium } \\
\text { dynamics equations (PDE) } \\
\text { (with BCS constitutive law) }\end{array}$ & $\begin{array}{l}\text { action potentials } \\
\text { FitzHugh-Nagumo-like models (PDE) }\end{array}$ \\
\hline
\end{tabular}

TABLE 1. The different scales of cardiac muscle contraction 


\section{On the molecular scale}

The myosin and actin molecules behave as nanomotors controlled by the neighboring Calcium ions. Motion generation is due to the coupling between thermal agitation and the chemical cycle of ATP hydrolysis. Myosin heads are subject to oscillations between a free state and an actin-bound state which makes them operate as an hybrid system. This situation can be modeled by controlled Langevin equations [14].

\section{On the sarcomere scale}

Actin and myosin molecules are respectively lined up on thin and thick filaments. The relative sliding of these filaments over one another is responsible for the changes in the sarcomere strain during the cardiac cycle. This "sliding-filament" assumption is well described by Huxley-like models [13]. The control of sliding is related to the changes in the transmembrane potential due to several ionic currents. Luo-Rudy-like models [16] account for a large number of ionic currents and can be used as models for the control on this scale.

\section{On the myofiber scale}

The lining-up of the sarcomeres results in the fibered structure of the myocytes, which constitute the contractile elements at the source of motion. Their collective behavior can be modeled by the BCS model [4]:

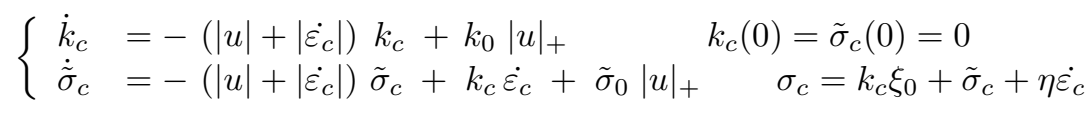

with $k_{c}$ the stiffness, $\sigma_{c}$ the stress, and $\varepsilon_{c}$ the strain of this contractile element, and $u$ the action potential. The relation between stress and strain is thus described by a set of ODE of visco-elasto-plastic type, subject to an electric control variable corresponding to the action potential. The changes in $u$ are ruled by FitzHughNagumo-like equations [8]. For sake of parameter identification, Luo-Rudy like models are indeed untractable on this scale as they include a very large number of unknown parameters.

\section{On the myocardium scale}

To account for the biomechanical behavior of the whole myocardium, the constitutive law (1) is embedded into a $3 \mathrm{D}$ anatomical model where it is used in the fiber directions and coupled with the dynamics equations. The electric control corresponds to the propagation of action potential and is ruled by FitzHugh-Nagumo-like reaction-diffusion equations.

It is worth noticing that, while we are well ahead in the multi-scale modeling of cardiac biomechanical behavior (see [4]), the corresponding modeling approach for the electric behavior remains to be done.

\section{Anatomical Mesh}

To carry on the computations of our model we need data regarding both the 3D ventricular geometry and the muscle fiber directions. Indeed, the anisotropy created by these fibers intervenes in both the electrical wave propagation and the mechanical contraction. There are different ways to obtain these fiber directions. We are currently using data from a dissected canine heart available from the Bioengineering Research Group ${ }^{2}$ of the University of Auckland, New Zealand and from reduced-encoding MR diffusion tensor imaging (dtMRI) [12].

In order to complete our anatomical model we also need data about the electrical network: the Purkinje network locations determine the electrical onset areas of the ventricular depolarization. But they are difficult to locate, both in dissection and cardiac images. In our model, they are currently approximated by a set of nodes near the apex.

\section{Electrical Wave Propagation}

The electrical behavior of the heartfrom, from the cell to the muscle level, has been extensively studied.

\footnotetext{
${ }^{2}$ http://www.bioeng. auckland.ac.nz/home/home.php
} 

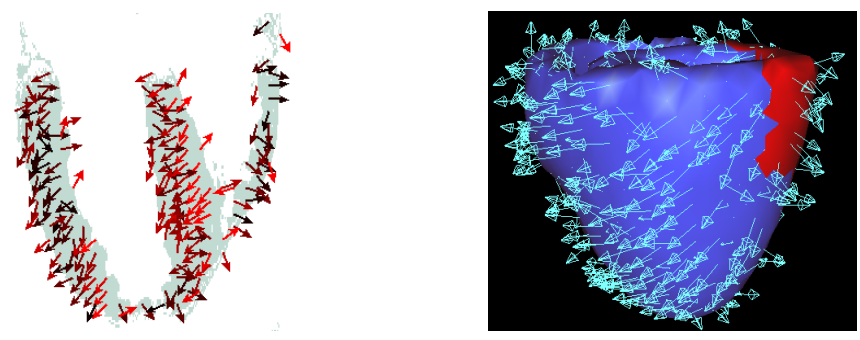

FIGURE 2. dtMRI slice and tetrahedral myocardium mesh built, with the fiber directions shown.

\section{Cell Level}

At the cell level, the main idea is to study the relationship between the transmembrane ionic currents and the ionic potentials inside and outside the cell. The models concerning this relation improve while the number of phenomena observed on the cell level increases [2]. At the beginning, we are only concerned with a model expected to account for the most important biological phenomena: a cell is activated only for a stimulus larger than a certain threshold; the shape of the action potential does not depend on the stimulus (it is only modeldependent); there is a refractory period during which the cell cannot be excited; a cell can act as a pacemaker.

A FitzHugh like model [8] seems to correctly capture these behaviors, and yields fast 3D computations. Here only the following set of differential equation is studied:

$$
\begin{aligned}
& \dot{u}=f(u)-z \\
& \dot{z}=\epsilon(\lambda u-z)
\end{aligned}
$$

where $f(u)=u(1-u)(u-a) . u$ is a normalized potential, $z$ is a dynamic variable modeling the repolarization ( $\epsilon$ and $\lambda$ are related to the repolarization rate and the repolarization decay).

\section{Whole Ventricle Level - Anisotropy}

At the macroscopic scale, the ventricles are considered as a conducting continuum, where the local potentials are undergoing at the same time the diffusion and the reaction phenomena described by the models above. Hence, (2) becomes:

$$
\begin{aligned}
& \dot{u}=\operatorname{div}(D \nabla(u))+f(u)-z \\
& \dot{z}=\epsilon(\lambda u-z) .
\end{aligned}
$$

On a physiological point of view, these equations are understood either as a mathematical approximation of the dynamical system introduced by Hodgkin and Huxley [11], as in [8], or as the result of some equilibrium equations that govern the conducting continuum, like in the so-called bidomain model [24].

The anisotropy of the ventricles is taken into account through the diffusion tensor $D: D=d_{0} \cdot \operatorname{diag}(1, \rho, \rho)$, in a local orthonormal basis $(\mathbf{i}, \mathbf{j}, \mathbf{k})$ where $\mathbf{i}$ is parallel to the fiber. $d_{0}$ is a scalar conductivity and $\rho$ the anisotropy ratio between the transverse and the axial conductivities.

\section{Results of the wave propagation}

Simulated isochrones of activation are presented (Fig. 3), after a wave was initialized at the apex, using a crude approximation of the Purkinje network and a slightly anisotropic diffusion tensor.

We can simulate different singularities that may correspond to pathologies by changing the conduction parameters (Fig. 4), for instance introducing a strong conductivity anisotropy.

This time-dependent computed potential can then be used as an excitation entry to the system describing the mechanical behavior of the myocardium. 

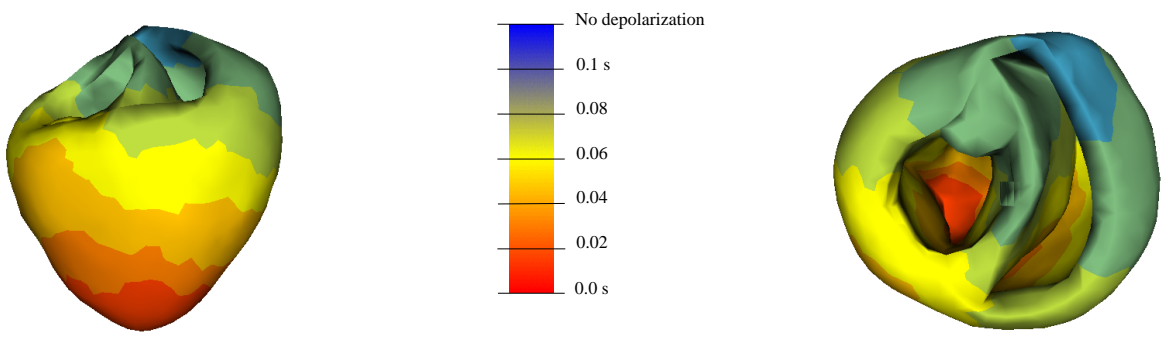

FIGURE 3. Isochrones of activation (computed with a slightly anisotropic diffusion tensor: $\rho=0.7$ )
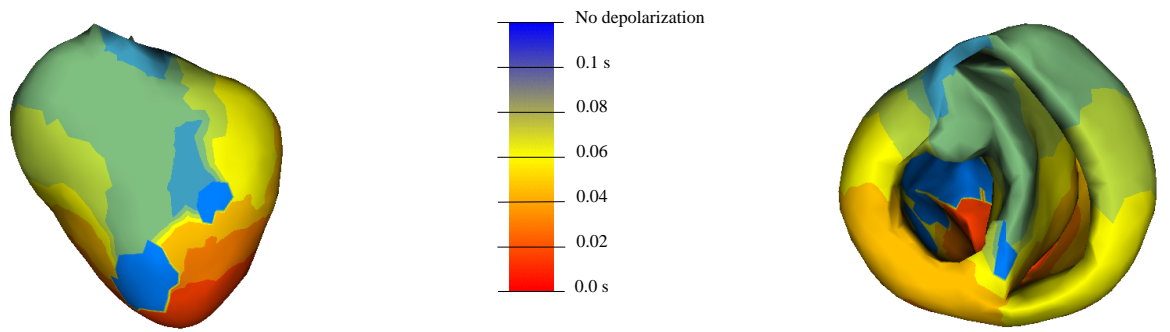

FIgURE 4. Apparitions of activation singularities with a highly anisotropic diffusion tensor.

\section{Electromechanical Coupling}

\subsection{Modeling}

\section{Formulation}

The mesoscopic myofiber constitutive law previously introduced (see also [4]) is now incorporated in a macroscopic rheological model of Hill-Maxwell type [6,10], as depicted in Figure 5. The element $E_{c}$ accounts for the contractile electrically-activated part of the behavior while elastic material laws are used for the series element $E_{s}$ and for the parallel element $E_{p}$. Based on experimental results, the corresponding stress-strain laws are generally assumed to be of exponential type [19].

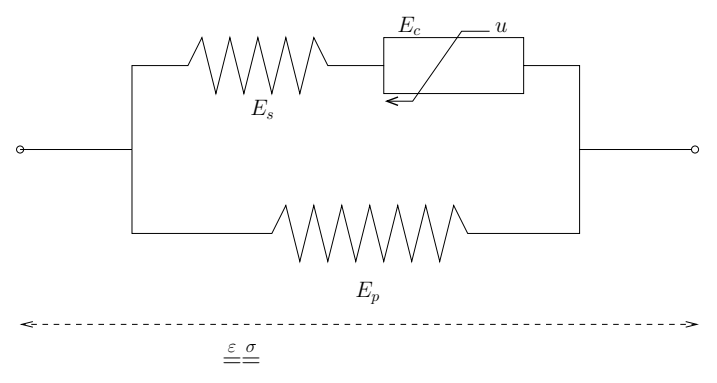

Figure 5. Hill-Maxwell rheological model.

The corresponding governing macroscopic three-dimensional (3D) mechanical equations are presented in [6] and we focus here on a 1D formulation of activated fiber contraction. The addition of viscous damping ( $\mu$ and 
$C)$ in parallel with element $E_{c}$ and element $E_{p}$ leads to the following formulation:

$$
\left\{\begin{array}{l}
\rho \ddot{y}-\frac{\partial}{\partial x}\left(k_{p}(\varepsilon)+C \dot{\varepsilon}+\sigma_{c}+\mu \dot{\varepsilon}_{c}+k_{c} \xi_{0}\right)=0 \\
\dot{\sigma}_{c}=k_{c} \dot{\varepsilon}_{c}-\left(\alpha\left|\dot{\varepsilon}_{c}\right|+|u|\right) \sigma_{c}+\sigma_{0}|u|_{+} \\
\dot{k}_{c}=-\left(\alpha\left|\dot{\varepsilon}_{c}\right|+|u|\right) k_{c}+k_{0}|u|_{+} \\
\sigma_{c}+\mu \dot{\varepsilon}_{c}+k_{c} \xi_{0}=k_{s}\left(\varepsilon-\varepsilon_{c}\right)
\end{array}\right.
$$

with appropriate initial conditions. In these equations, $\varepsilon$ denotes $\frac{d y}{d x}$ and the indices $s, c$, and $p$ refer to the series, contractile and parallel elements, respectively, with $k$ the stiffnesses. The successive phases of the cardiac cycle are distinguished in the boundary conditions.

\section{Simulations}

Simulations of problem (4) were carried out taking into account the four phases of a cardiac cycle (isovolumetric contraction, ejection, isovolumetric relaxation and filling). The results obtained - especially the evolution of stresses and strains - correspond reasonably well to heart physiology. Figures 6(a) and 6(b) present the displacement and stress variations during a cardiac cycle.

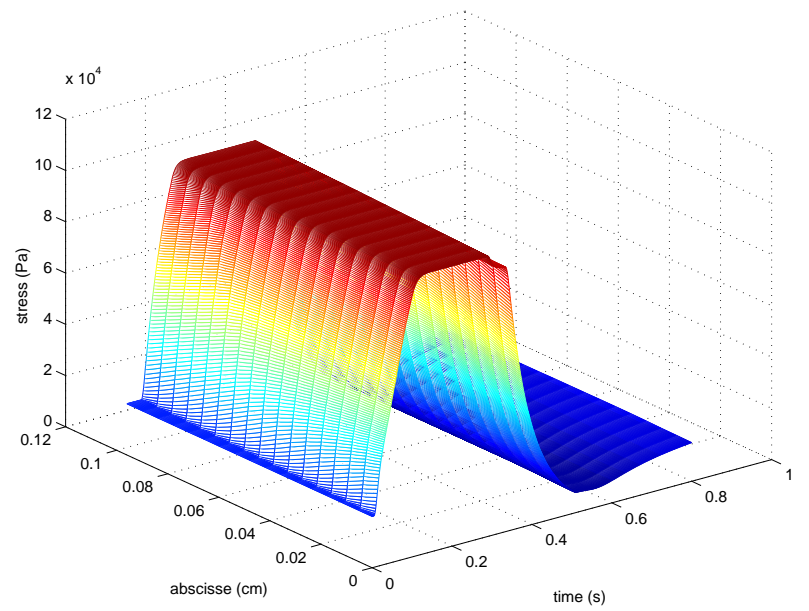

(a) Evolution of stress in a fiber along a cycle.

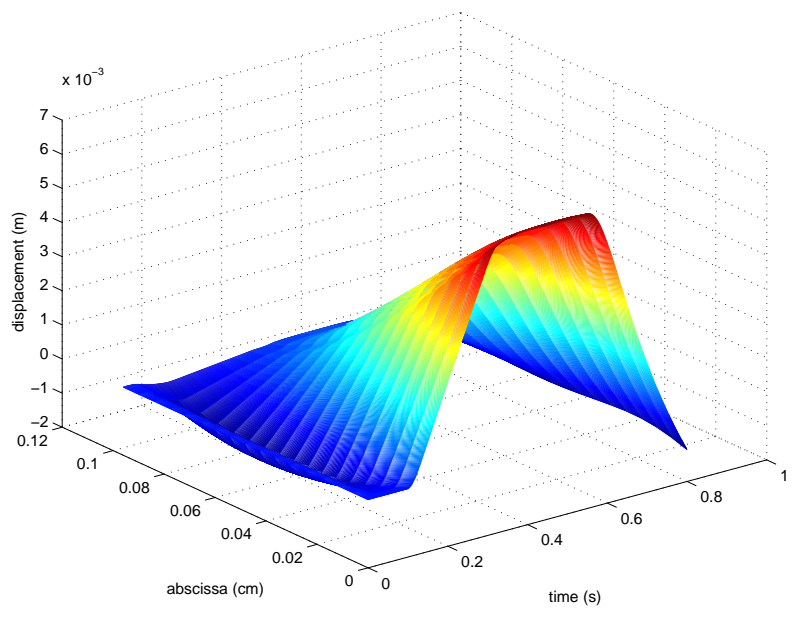

(b) Displacement of a fiber along a cycle.

FIGURE 6. Displacement and stress variations.

\subsection{Data assimilation}

Mechanical phenomena in the heart are nonlinear, fast and with large strains so a complete modeling is very difficult to deal with. Furthermore, measurements of cardiac activity (blood pressure, strains and displacements of ventricle walls,...) are scattered and noisy. Hence, our objective is to use a data assimilation approach in order to identify the electromechanical model using the available measurements. The problem could be formulated as follows : find the parameters $\bar{\sigma}_{0}, \bar{k}_{0}$ and the input $\bar{u}(x, t)$ such that the state function $X=$ $\left(y, \dot{y}, \sigma_{c}, k_{c}, \varepsilon_{c}\right)^{t}$, solution of problem (4) satisfies $Y\left(t_{k}\right)=H X\left(t_{k}\right)$ for all $k \in I$ where $\left\{Y\left(t_{k}\right)\right\}_{k \in I}$ is the set of available measurements and $H$ the observation operator. The sequential algorithm employed to achieve this state-parameter estimation is based on a Kalman filtering approach using the SEEK formulation $[5,7]$.

We give below examples of estimations carried out with numerically simulated observations $\left\{Y\left(t_{k}\right)\right\}_{k \in I}$ obtained as follows. The solution $X$ of problem (4) with given parameters $\bar{\sigma}_{0}, \bar{k}_{0}$ and a given input $\bar{u}(x, t)$ is 
simulated over the time interval $[0, T]$. Then observations $\left\{Y\left(t_{k}\right)\right\}_{k \in I}$ are obtained by $Y\left(t_{k}\right)=H X\left(t_{k}\right)$, where $H=\left[\begin{array}{lllll}1 & 0 & 0 & 0 & 0\end{array}\right]$ and $t_{k}$ are samples chosen in $[0, T]$. Finally, from the observation data $\left\{Y\left(t_{k}\right)\right\}_{k \in I}$ and the model (4) where the initial values of parameters $\tilde{\sigma}_{0}, \tilde{k}_{0}$ and input $\tilde{u}(x, t)$ are selected with $\left(\tilde{\sigma}_{0}, \tilde{k}_{0}, \tilde{u}\right) \neq\left(\bar{\sigma}_{0}, \bar{k}_{0}, \bar{u}\right)$ (taking as initial values $\left(\tilde{\sigma}_{0}, \tilde{k}_{0}, \tilde{u}\right)$ roughly half of the correct ones), we obtain an estimation of the correct values $\bar{\sigma}_{0}, \bar{k}_{0}$ and $\bar{u}(x, t)$, see Figures $7(\mathrm{a})$ and $7(\mathrm{~b})$.

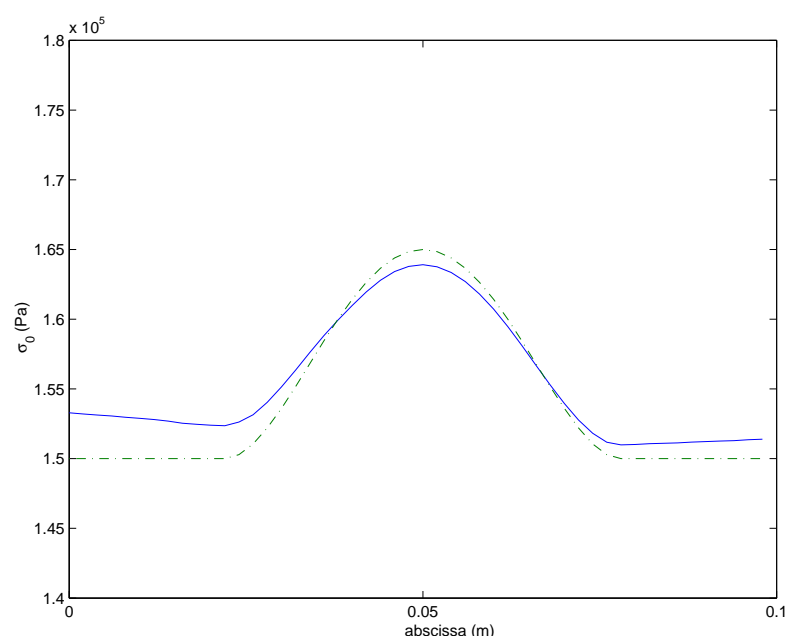

(a) Value of $\bar{\sigma}_{0}$ and its estimation.

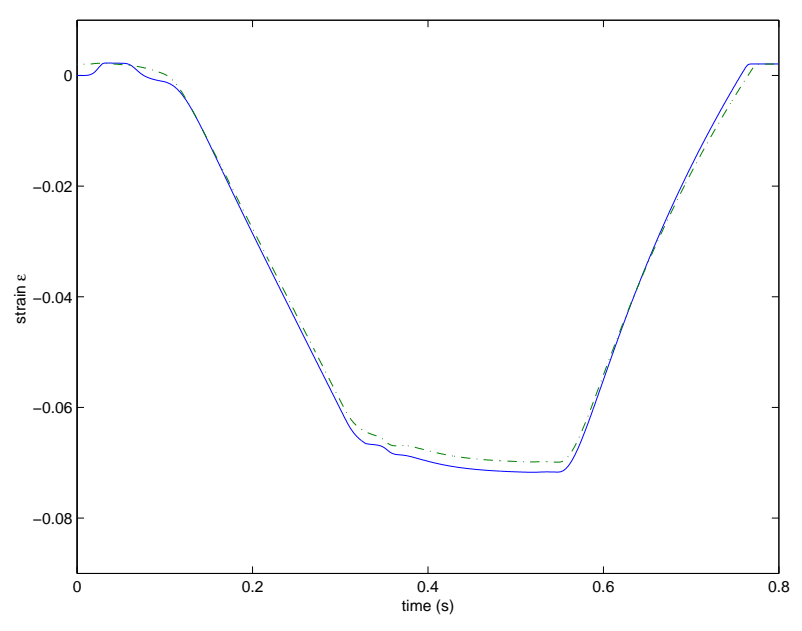

(b) Strain $\varepsilon\left(x_{0}, t\right)$ simulated with model (4) vs. $\left(\bar{\sigma}_{0}, \bar{k}_{0}, \bar{u}\right)$ and its estimation with the procedure detailed in 4.2 .

Figure 7. Comparison between the simulation $(-\cdot-)$ and the estimation $(-)$ of different parameters.

We observe that the results of this inverse problem (parameters and input determination) are quite good. The $3 \mathrm{D}$ case is under current research.

\section{Interaction with Cardiac Images}

Thus, to adjust the parameters of the model, we need to extract the cardiac motion from cardiac images, in order to compare these measures with the computation results. Many different techniques were used in cardiac image analysis with 3D models [9]. In the deformable model field, some of the ventricular function parameters (ventricles volume, wall thickness,...) can be efficiently extracted from the deformation of geometric surfaces. But these surfaces do not include any biological or physical a priori knowledge to guide their deformations where boundary data is missing. Moreover, only the apparent motion (ie. displacement along the normal direction) can be reconstructed. Recently, volumetric models have been used [21,22], they have a strong topology constraint and they can easily include a priori information [15]. Moreover, there is a twist during contraction in the heart motion, using biomechanical volumetric models could help recover this tangential displacement.

In the deformable model framework, a model evolves under the influence of two energies: an External Energy which makes the model fit the images and an Internal Energy which acts as a regularization term and can include a priori information (shape, physical properties, motion,...). In our approach, the computation of this External Energy at a surface vertex depends not only on the vertex location but also on its normal direction. Different type of forces may be applied depending on the image modality. We chose to combine intensity and gradient information with a region-based approach [20] applied to the intensity profile extracted at each vertex in its normal direction. It consists in defining a region with a range of intensity values and then finding its 

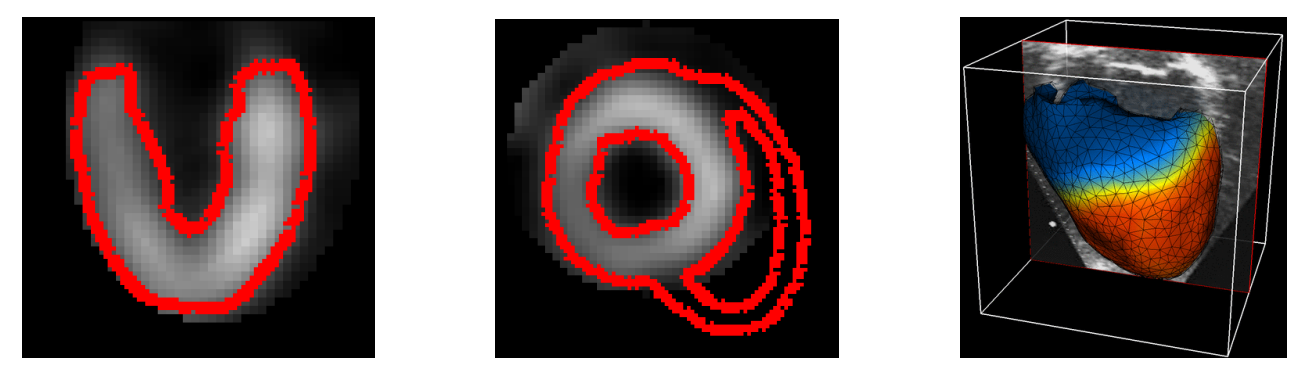

FIGURE 8. Segmentation of cardiac images with a volumetric biomechanical model and simplified electromechanical model in a $4 \mathrm{D}$ ultrasound image

boundary by looking at the voxels of high gradient value. The extent of the intensity profile is decreased in the coarse-to-fine process. Then, we apply a force $F_{i}$ which is proportional to the distance to the closest boundary point of the image from the considered point of the mesh surface. The volumetric nature of our model strongly decreases the importance of the image outliers in the motion estimation since it strongly constrains the geometric (for instance the thickness of the myocardium wall) and physical behavior. The Internal Energy corresponds to a simplified version of the electromechanical model described in the previous sections: to allow an interactively controlled segmentation, we need a computationally fast model ${ }^{3}$. Images and videos are available on the web ${ }^{4}$

\section{Simplified Electromechanical Model}

The qualitative behavior of the electromechanical coupling is a contraction for a positive action potential and an active relaxation for a negative one. Moreover, the action potential also modifies the stiffness of the material. The model introduced in $[3,4]$ by Bestel, Clment and Sorine and presented in the previous sections captures this behavior. For computational efficiency, we simplified this physical model by using a piecewise-linear anisotropic material for the stiffness $k$, and only an electrical command for contraction stress tensor $\sigma_{c}$ (Fig. 9).

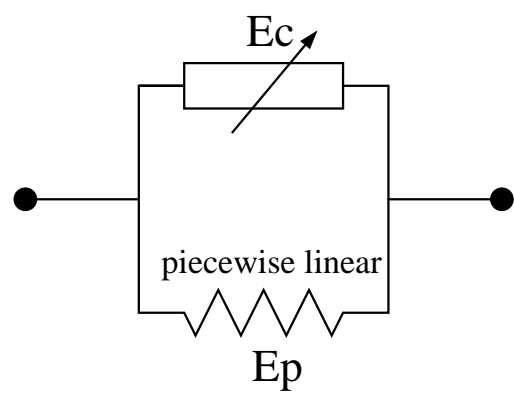

Figure 9. Simplified rheological model.

The simplified coupling equation from (1) writes: $\dot{\sigma_{c}}=-|u| \sigma_{c}+\sigma_{0}|u|_{+}$. It only takes into account the electrical command. The contraction stress increases exponentially for positive action potential and decreases exponentially for negative ones, and the variation rate depends on the action potential value (Fig. 10).

We obtained preliminary results with this simplified electromechanical model, we now have to adjust the electrical and the coupling parameters to a patient dataset, to be able to use it to segment a whole cardiac sequence.

\footnotetext{
${ }^{3}$ An earlier version of this work was published in [23]

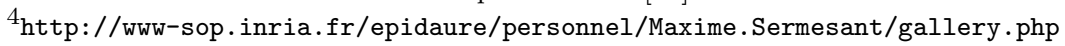




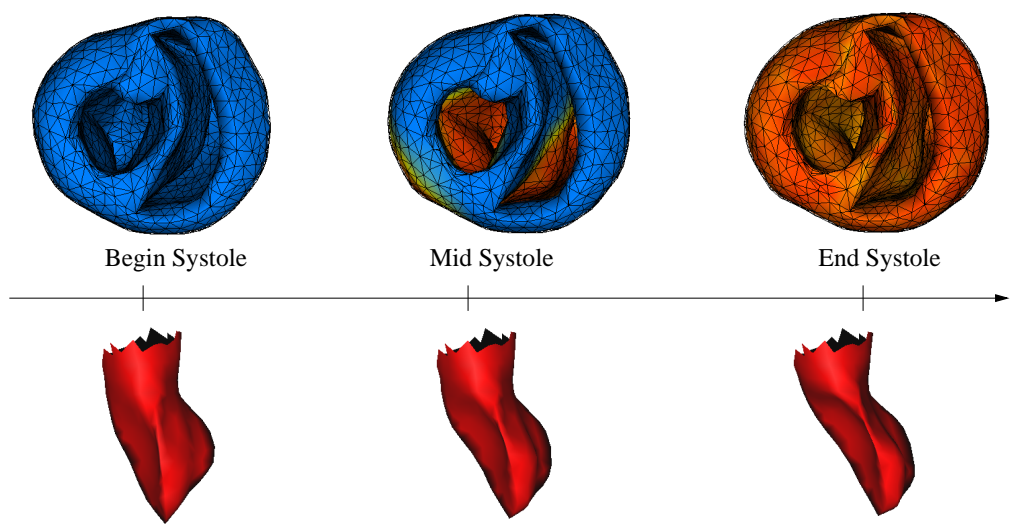

Figure 10. Contraction of the fibers induced by the simplified electromechanical coupling, and deformation of the left ventricle.

\section{Conclusion and Perspectives}

Each part of our model is planned to be improved:

- the anatomical model should be adapted to a human heart. Diffusion tensor MRI is probably one possible way to obtain human fiber directions;

- the electrical model should include a third variable to better control the shape of the wave and a mechano-electrical feedback;

- the complex mechanical model has to be solved on a 3D mesh;

- the simplified model for image segmentation will become non-linear and later integrate a series element.

For each of these models we intend to identify parameters:

- the electrical parameters and the electrical entries, should be estimated by comparing computed ECGs with measured ones. To achieve this, an inverse problem has to be considered;

- for the mechanical parameters, identification techniques still have to be developed, as in vivo rheological studies for human tissues are hard to set up. Here, a criterion will be the difference between the computed motion and the one extracted from the cardiac images.

All these points will be the topics of our future work. Globally, recent measurements of the electrical activity, fiber directions and motion reconstruction (from tagged MRI) on the same heart should help adjust the different parameters of this model [18].

\section{REFERENCES}

[1] N. Ayache, D. Chapelle, F. Clément, Y. Coudière, H. Delingette, J.A. Désidéri, M. Sermesant, M. Sorine, and J. Urquiza. Towards model-based estimation of the cardiac electro-mechanical activity from ECG signals and ultrasound images. In T. Katila, I. Magnin, P. Clarysse, J. Montagnat, and J. Nenonen, editors, Functional Imaging and Modeling of the Heart (FIMH'01), number 2230 in Lecture Notes in Computer Science (LNCS), pages 120-127. Springer, 2001.

[2] A. L. Bardou, P. M. Auger, P. J. Birkui, and J.-L. Chassé. Modeling of cardiac electrophysiological mechanisms: From action potential genesis to its propagation in myocardium. Critical Reviews in Biomedical Engineering, 24:141-221, 1996.

[3] J. Bestel. Modèle différentiel de la contraction musculaire contrôlée : Application au système cardio-vasculaire. PhD thesis, Université Paris 9, 2000.

[4] J. Bestel, F. Clément, and M. Sorine. A biomechanical model of muscle contraction. In W.J. Niessen and M.A. Viergever, editors, Medical Image Computing and Computer-Assisted intervention (MICCAI'01), volume 2208 of Lecture Notes in Computer Science (LNCS), pages 1159-1161. Springer, 2001.

[5] M.A. Cane, A. Kaplan, N. Miller, B. Tang, Hackert E.C., and Busalacchi A.J. Mapping tropical pacific sea level : Data assimilation via a reduced state Kalman filter. J. Geophys. Res., 101(2):599-617, 1996. 
[6] D. Chapelle, F. Clément, F. Génot, P. Le Tallec, M. Sorine, and J. Urquiza. A physiologically-based model for the active cardiac muscle contraction. In T. Katila, I. Magnin, P. Clarysse, J. Montagnat, and J. Nenonen, editors, Functional Imaging and Modeling of the Heart (FIMH'01), number 2230 in Lecture Notes in Computer Science (LNCS), pages 128-133. Springer, 2001.

[7] Pham D.T., J. Verron, and Roubaud M.C. Singular evolutive Kalman filter with EOF initialization for data assimilation in oceanography. J. Mar. Syst., 16:323-340, 1997.

[8] R.A. FitzHugh. Impulses and physiological states in theoretical models of nerve membran. Biophys. J., 1:445-466, 1961.

[9] A.F. Frangi, W.J. Niessen, and M.A. Viergever. Three-dimensional modeling for functional analysis of cardiac images: A review. IEEE Trans. on Medical Imaging, 1(20):2-25, 2001.

[10] A.V. Hill. The heat of shortening and the dynamic constants in muscle. Proc. Roy. Soc. London (B), 126:136-195, 1938.

[11] A.L. Hodgkin and A.F. Huxley. A quantitative description of membrane current and its application to conduction and excitation in nerve. J. Physiol, 177:500-544, 1952.

[12] E.W. Hsu and C.S. Henriquez. Myocardial fiber orientation mapping using reduced encoding diffusion tensor imaging. Journal of Cardiovascular Magnetic Resonance, 3:325-333, 2001.

[13] A.F. Huxley. Muscle structure and theories of contraction. Progress in biophysics and biological chemistry, 7:255-318, 1957.

[14] F. Jülicher, A. Ajdari, and J. Prost. Modeling molecular motors. Reviews of Modern Physics, 69, October 1997.

[15] W-T. Lin and R.A. Robb. Simulation and interactive multi-dimensional visualization of cardiac dynamics using a patientspecific physics-based model. In Computer Assisted Radiology and Surgery (CARS'00), 2000.

[16] C.H. Luo and Y. Rudy. A model of the ventricular cardiac action potential: depolarization, repolarization, and their interaction. Circ. Res., 68:1501-1526, 1991.

[17] A. McCulloch, J.B. Bassingthwaighte, P.J. Hunter, D. Noble, T.L. Blundell, and T. Pawson. Computational biology of the heart: From structure to function. Progress in Biophysics \& Molecular Biology, 69(2/3):151-559, 1998.

[18] E. McVeigh, O. Faris, D. Ennis, P. Helm, and F. Evans. Measurement of ventricular wall motion, epicardial electrical mapping, and myocardial fiber angles in the same heart. In T. Katila, I. Magnin, P. Clarysse, J. Montagnat, and J. Nenonen, editors, Functional Imaging and Modeling of the Heart (FIMH'01), number 2230 in Lecture Notes in Computer Science (LNCS), pages 76-82. Springer, 2001.

[19] I. Mirsky and W.W. Parmley. Assessment of passive elastic stiffness for isolated heart muscle and the intact heart. Circul. Research, 33:233-243, 1973.

[20] J. Montagnat, M. Sermesant, H. Delingette, G. Malandain, and N. Ayache. Anisotropic filtering for model-based segmentation of 4D cylindrical echocardiographic images. Pattern Recognition Letters, 24:815-828, 2003.

[21] X. Papademetris, A. J. Sinusas, D. P. Dione, and J. S. Duncan. Estimation of 3D left ventricle deformation from echocardiography. Medical Image Analysis, 5(1):17-28, 2001.

[22] Q.C. Pham, F. Vincent, P. Clarysse, P. Croisille, and I. Magnin. A FEM-based deformable model for the 3D segmentation and tracking of the heart in cardiac MRI. In Image and Signal Processing and Analysis (ISPA'01), 2001.

[23] M. Sermesant, Y. Coudière, H. Delingette, N. Ayache, and J.A. Désidéri. An electro-mechanical model of the heart for cardiac image analysis. In W.J. Niessen and M.A. Viergever, editors, Medical Image Computing and Computer-Assisted Intervention (MICCAI'01), volume 2208 of Lecture Notes in Computer Science (LNCS), pages 224-231. Springer, 2001.

[24] K. Simelius, J. Nenonen, and B.M. Horácek. Simulation of anisotropic propagation in the myocardium with a hybrid bidomain model. In T. Katila, I. Magnin, P. Clarysse, J. Montagnat, and J. Nenonen, editors, Functional Imaging and Modeling of the Heart (FIMH'01), number 2230 in Lecture Notes in Computer Science (LNCS), pages 140-147. Springer, 2001. 